\title{
Optimisation of the performance of holographic beam-shaping diffractive diffusers through refinement of the recording process
}

Cara Jones

Technological University Dublin

Suzanne Martin

Technological University Dublin, suzanne.martin@tudublin.ie

Sanjay Keshri

Technological University Dublin, sanjaykumar.keshri@tudublin.ie

See next page for additional authors

Follow this and additional works at: https://arrow.tudublin.ie/cieocon2

Part of the Ophthalmology Commons, and the Optics Commons

\section{Recommended Citation}

Cara Jones, Suzanne Martin, Sanjay Keshri, Dervil Cody, Kevin Murphy, "Optimisation of the performance of holographic beam-shaping diffractive diffusers through refinement of the recording process," Proc. SPIE 11367, Photosensitive Materials and their Applications, 1136706 (13 April 2020); doi: 10.1117/ 12.2555774

This Conference Paper is brought to you for free and open access by the Centre for Industrial and Engineering Optics at ARROW@TU Dublin. It has been accepted for inclusion in Conference Papers by an authorized administrator of ARROW@TU Dublin. For more information, please contact arrow.admin@tudublin.ie, aisling.coyne@tudublin.ie,gerard.connolly@tudublin.ie. Funder: Enterprise Ireland

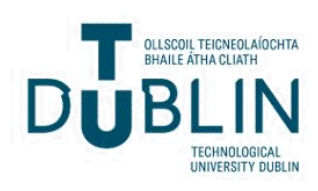




\section{Authors}

Cara Jones, Suzanne Martin, Sanjay Keshri, Dervil Cody, and Kevin Murphy 


\section{Optimisation of the performance of holographic beam-shaping diffractive diffusers through refinement of the recording process}

Jones, Cara, Martin, Suzanne, Keshri, Sanjay, Cody, Dervil, Murphy, Kevin

Cara Jones, Suzanne Martin, Sanjay Keshri, Dervil Cody, Kevin Murphy, "Optimisation of the performance of holographic beam-shaping diffractive diffusers through refinement of the recording process," Proc. SPIE 11367, Photosensitive Materials and their Applications, 1136706 (13 April 2020); doi: $10.1117 / 12.2555774$

SPIE. Event: SPIE Photonics Europe, 2020, Online Only 


\title{
Optimisation of the performance of holographic beam-shaping diffractive diffusers through refinement of the recording process
}

\author{
Cara Jones $^{\mathrm{a}}$, Suzanne Martin ${ }^{\mathrm{a}}$, Sanjay Keshri ${ }^{\mathrm{a}}$, Dervil Cody ${ }^{\mathrm{a}}$, and Kevin Murphy ${ }^{\mathrm{a}}$ \\ ${ }^{a}$ Centre for Industrial and Engineering Optics, School of Physics and Clinical and Optometric \\ Sciences, Technological University Dublin, Dublin, Ireland
}

\begin{abstract}
Optical diffusers have uses in laser applications and machine vision. Typical fabrication at a commercial level requires master production and the stamping/copying of individual elements at scale. This expensive, indirect process inhibits custom diffusers at reasonable cost. Previously the authors published a novel, direct, single beam method of recording customizable and controllable volume holographic diffusers by manipulating laser speckle and recording the pattern in photopolymer. This method allows for beam-shaping to produce diffusion patterns of various sizes and shapes. In this work, the direct method of recording controllable holographic diffusers is refined to improve diffuser performance (i.e., a decrease in zero order strength) for a simple diffuser. This is achieved through optimising the recording conditions (exposure energy, power and layer thickness) for a given photopolymer formulation. Significant improvement in the diffuser efficiency is observed through the optimisation process for a particular speckle size, resulting in a five-fold decrease in the remaining zero order. Kogelnik Coupled Wave Theory (KCWT) is explored as a first step towards developing an appropriate model for the behaviour of holographic elements recorded with interference patterns formed through stochastic processes, such as speckle patterns.
\end{abstract}

Keywords: Holography, Volume gratings, Diffusers, Photopolymer, Diffractive optics, Holographic optical elements

\section{INTRODUCTION}

Holography is a process which captures both the phase and intensity information of a wavefront propagating from an illuminated object typically by recording an interference pattern from two beams (a reference and an object beam) into a recording material. Commonly used recording materials include silver halide, dichromated gelatin (DCG) and photopolymer. ${ }^{1}$ The intensity and phase of the object beam can be retrieved by probing the hologram with a beam of light recreating the reference beam at the appropriate angle. ${ }^{2}$ Volume phase holograms are holograms where the recording of the photonic structure takes place entirely within the volume of the recording material, rather than by the creation of surface structures.

Optical diffusing elements have a variety of uses, particularly in laser applications and machine vision to reduce hotspots and produce certain beam shapes. Various optical elements were traditionally used as diffusers including ground glass and roughened plastic surfaces. The growing need for homogenous diffuse beam patterns in recent decades led to the development of engineered surfaces and surface holographic elements, ${ }^{3} .{ }^{4}$ For these type of elements to be fabricated at commercial quantities and costs they require the production of a master, through methods such as laser etching or lithography, which is typically expensive. This master is then stamped/copied into the final individual elements at scale. This is inherently an indirect process which inhibits availability of custom designed diffusers at reasonable cost.

In addition to surface elements, volume holographic diffusers have also been reported in the literature. These have been produced by creating an in-line hologram of a ground glass diffuser in a two-beam recording setup ${ }^{5}$

Further author information: (Send correspondence to K.M)

K.M.: E-mail: kevin.p.murphy@tudublin.ie

Photosensitive Materials and their Applications, edited by Robert R. McLeod, Inmaculada Pascual Villalobos,

Yasuo Tomita, John T. Sheridan, Proc. of SPIE Vol. 11367, 1136706 - () 2020 SPIE

CCC code: $0277-786 \mathrm{X} / 20 / \$ 21 \cdot$ doi: $10.1117 / 12.2555774$ 
and by directly contact-copying an optical diffuser into photopolymer. ${ }^{6}$ Neither of these methods offer control over the diffusivity or the shape of the diffusion pattern emanating from the hologram. Recently Scattering Optical Elements (SOEs), which do provide a level of control of the beam output, have also been fabricated and consist of a volume holographic photopolymer layer and a traditional diffusive layer. ${ }^{7}$ However, they are computationally exacting, require a known wavefront input and are not formed by a direct holographic method.

Previously the authors published, for the first time, a direct, single beam method of recording customizable and controllable volume holographic diffusers by manipulating laser speckle. ${ }^{8}$ This method allows for the beamshaping of the output to produce diffusion patterns of various sizes and shapes. This was carried out with an acrylamide-based photopolymer formulation as the recording material. ${ }^{9}$ This has many advantages in terms of mass-production as it requires no post-processing, is stable pre- and post-recording, records in the volume of the material, is reproducible and can be processed via roll-to-roll methods. ${ }^{10}$

In this paper we detail how the direct method of recording controllable holographic diffusers is refined to improve the performance of the diffusers (i.e. obtain a decrease in zero order strength). Section 2 provides the experimental methods used to optimise the recording conditions for fabricating diffusers in a given photopolymer formulation and test their effectiveness. Section 3 describes modelling of the performance of holographic elements recorded with interference patterns formed through stochastic processes such as speckle patterns using Kogelnik Coupled Wave Theory (KCWT); in addition, the relevance of this approach (i.e., the use of KCWT) for holographic diffusers is assessed. Section 4 presents the experimental results on diffuser performance, while Section 5 outlines the conclusions from the work.

\section{EXPERIMENTAL METHODS}

\subsection{Recording Setup, Conditions and Materials}

The fabrication of the customisable volume holographic diffusers is underpinned by speckle theory, in particular that which deals with subjective speckle. In this case a speckle pattern, which is produced by randomising the phase of a beam (e.g by a rough surface), is imaged by a lens before illuminating an area. This means the speckle size displayed in the focal plane of the lens depends on the not only the wavelength and the diameter of the illuminated area but also on the (finite) lens aperture. The minimum size of speckle in the subjective speckle case, $\sigma$, is governed by the following equation;

$$
\sigma=\frac{(2.44 \lambda f)}{a}
$$

where $f$ is the focal length of the focusing lens, $a$ its aperture diameter and $\lambda$ the wavelength of light.

Precise control of the range of speckle sizes found in the lens focal plane can be achieved through controlling the lens pupil diameter using a variable aperture. Placing a photopolymer layer at the focal plane will result in the speckle pattern being recorded holographically in the photopolymer volume. Varying the speckle size will vary the feature size in the photopolymer, in turn adjusting the level of diffraction through the material.

The holographic recording setup for the diffusers is essentially the same as described by the authors previously ${ }^{8}$ and is shown in Fig. 1 above. The main difference from that described previously is that the aperture is kept at a constant size so that the average speckle size remains the same. This is done so that the recording conditions can be accurately optimised, as there may be a different optimum for each speckle/feature size.

In this work the aperture diameter is kept constant at $12 \mathrm{~mm}$ and $f_{f l}=15 \mathrm{~cm}$, which provides an average speckle size of $15.7 \mu \mathrm{m}$ in the speckle pattern recorded. This speckle size is within the range that has previously been accurately produced and recorded in the photopolymer layer. ${ }^{8}$ Three different recording intensities are studied $\left(0.75 \mathrm{~mW} / \mathrm{cm}^{2}, 1 \mathrm{~mW} / \mathrm{cm}^{2}\right.$ and $\left.1.25 \mathrm{~mW} / \mathrm{cm}^{2}\right)$ for a range of exposure times $(30-180 \mathrm{~s})$ to find the optimum conditions for recording diffusers. Three different photopolymer layer thickness are studied $(\approx 50 \mu \mathrm{m}$, $70 \mu \mathrm{m}, 100 \mu \mathrm{m}, \pm 5 \mu \mathrm{m})$ by depositing different volumes of photopolymer solution $(0.5 \mathrm{~mL}, 0.75 \mathrm{~mL}$ and $1 \mathrm{~mL})$ on a standard glass microscope slide. These layers were covered with a $50 \mu \mathrm{m}$ film laminated on top to prevent the formation of surface structures during polymerisation. The photopolymer formulation used is an acrylamide based photopolymer similar to that described by Martin et al. ${ }^{9}$ The base constituents are a $10 \% \mathrm{w} / \mathrm{w}$ PVA stock solution $(17 \mathrm{~mL})$, acrylamide $(0.8 \mathrm{~g})$ and bis-acrylamide $(0.2 \mathrm{~g})$ monomers, triethanolamine (TEA) $(2 \mathrm{~mL})$ as an electron donor and plasticiser and $4 \mathrm{~mL}$ Erythrosine B stock dye solution (0.11g in 100mL deionised water). 


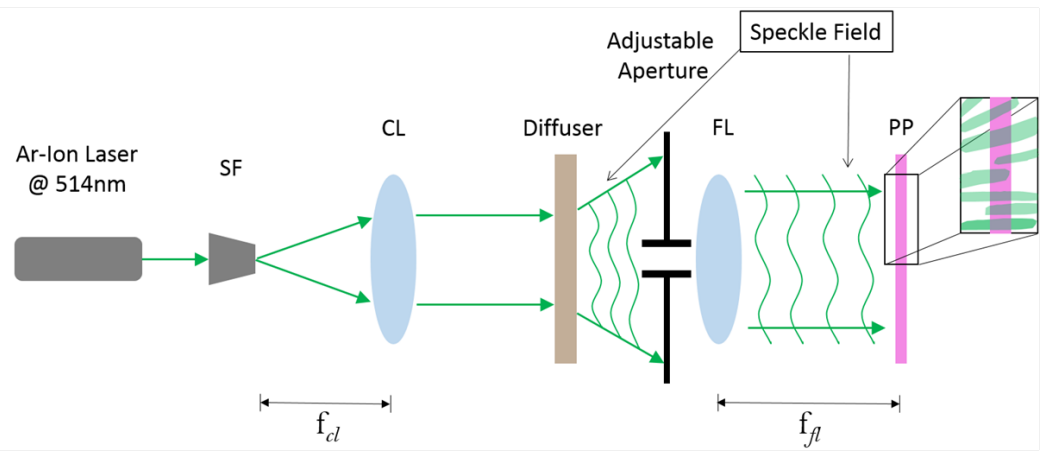

Figure 1: Setup schematic for recording laser speckle diffusers in photopolymer. SF = Spatial Filter, CL $=$ Collimating Lens, $f_{c l}=$ focal length of CL, FL $=$ Focusing Lens, $f_{f l}=$ focal length of the FL, PP $=$ Photopolymer Layer.

\subsection{Probing Setups}

To characterise the strength of the diffusers a test system has been developed. This system probes the diffusers with a HeNe laser $(633 \mathrm{~nm})$ over a range of incidence angles and the power in the zero order is measured by a power meter, with an $\approx 2 \mathrm{~mm}$ aperture placed over it (see Fig. 2a). The strength of the diffuser can be referred to as the diffuser efficiency. This diffuser efficiency factor is analogous to the haze factor and is defined by Eq.2. The control zero order intensity is obtained by measuring the power of a beam transmitted through a control sample of a bleached photopolymer layer, with no photonic structure recorded in it.

$$
\text { Diffuser Eff. }=\left(1-\frac{\text { Zero Order Intensity Diffused }}{\text { Zero Order Intensity Control }}\right) \times 100 \%
$$

In addition a system was assembled to quantify the spread of the light transmitted through the diffuser in the far field. This system is used to construct a beam output map and is illustrated in Fig. 2b. In this case both the probe source (laser diode $532 \mathrm{~nm}$ ) and the diffuser are placed on a rotation stage and rotated together through a range of angles. The transmitted light from that output angle is detected by a power meter with an aperture $(\approx 2 \mathrm{~mm})$ placed over it. The power meter is held at a distance of $\approx 11 \mathrm{~cm}$ from the sample.

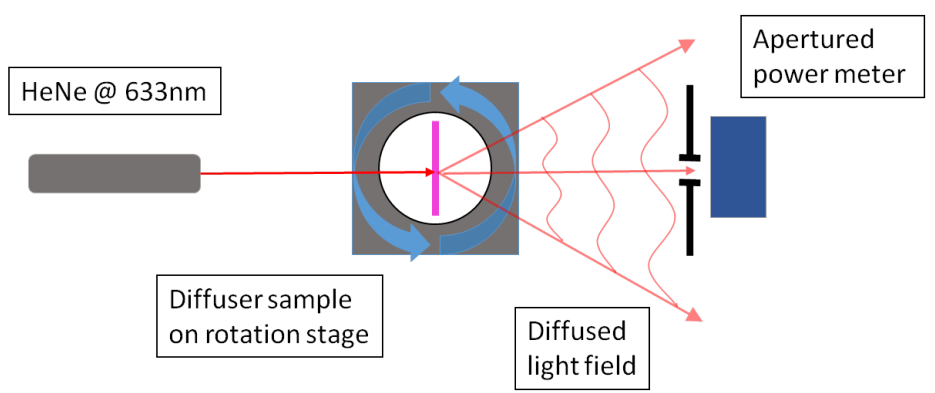

(a)

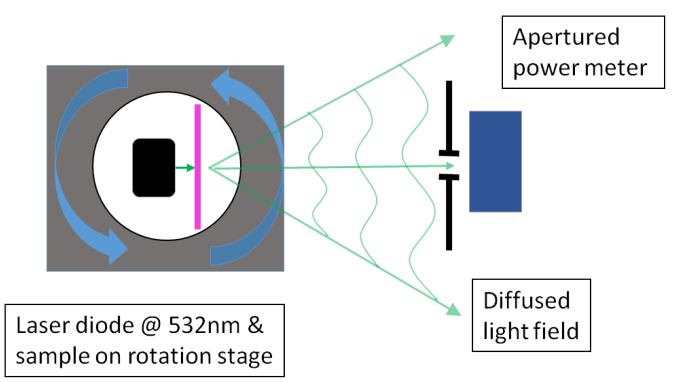

(b)

Figure 2: Setup for measuring the diffuser efficiency of the samples (a) and setup for determining the beam output map of the diffusers (b).

\section{MODELLING OF DIFFUSER PERFORMANCE}

\subsection{Modelling of Q Parameter}

The holographic diffusers are a complex system as they are naturally made up of a stochastic speckle pattern, defined in theory through probabilities. In addition to this the act of holographic recording them as a type of diffraction grating adds another layer of complexity. Therefore modelling the diffraction of light through 
such structures is not straightforward. In spite of these factors an attempt is made here to assess the potential suitability a of standard method to approximate the diffraction through a holographic diffuser.

Kolgelnik Coupled Wave Theory $(\mathrm{KCWT})^{11}$ is frequently used to model diffraction gratings. ${ }^{12} \mathrm{KCWT}$ is devised to be used when gratings are in what is referred to as the "thick" regime. This is defined as the regime in which a single diffracted order is observed, rather than many orders, and diffraction efficiency approaching $100 \%$ is possible. The Q parameter is often used to define the "thin" and "thick" regimes where $Q<1=$ "thin" regime, $Q>10=$ "thick" regime and $10>Q>1=$ intermediate of mixed regime. The Q parameter itself is defined by Eq. (3)

$$
Q=\frac{2 \pi \lambda d}{n \Lambda}
$$

where, $\lambda=$ wavelength of the illuminating beam, $d=$ thickness of recording medium, $n=$ average refractive index of the medium, $\Lambda=$ grating spacing.

The Q parameter has been calculated for the relevant thickness range used in this work $(45 \mu \mathrm{m}-105 \mu \mathrm{m})$, with $\lambda=633 \mathrm{~nm}$ and $n=1.5$ as constants. The spatial frequency range used is centred on $64 \mathrm{lines} / \mathrm{mm}$, which is obtained based on using the speckle size $(\sigma=15.7 \mu \mathrm{m})$ as a surrogate for the spatial period. The results of these calculations are seen in Fig. (3). These indicate that for majority of the diffusers recorded Q borders on 1 , which is on the limit of the "thin" regime. This would imply that KCWT will not be ideal (or perhaps even useful) in modelling the diffraction from the diffusers. However, since very high diffuser efficiencies have been observed in these elements KCWT is worth exploring as a first step.

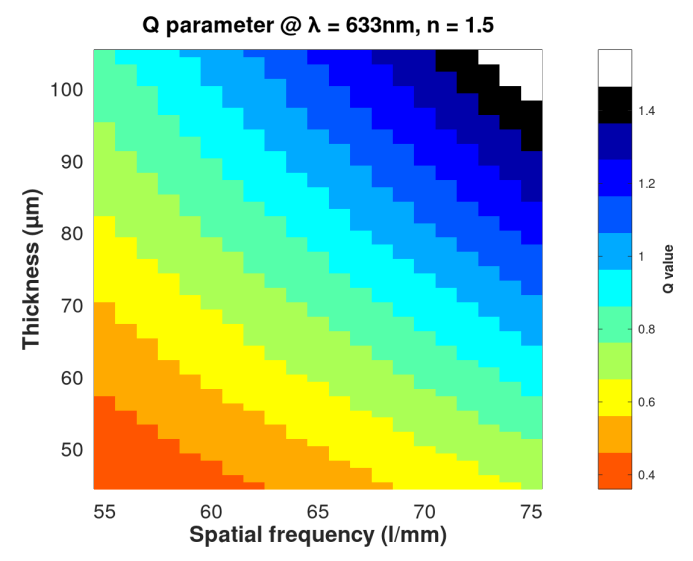

Figure 3: Calculation of the Q parameter for thickness range of $45 \mu \mathrm{m}-105 \mu \mathrm{m}$ and spatial frequency $=55-75$ lines $/ \mathrm{mm}$.

\subsection{Modelling of Angular Selectivity Curves}

In order to examine whether KCWT has any potential in modelling the diffraction output for these volume holographic diffusers a set of angular selectivity curves were calculated using KCWT. The parameters used for these calculations were $\lambda=633 \mathrm{~nm}$, spatial frequency $=64$ lines $/ \mathrm{mm}$ and a peak diffraction efficiency was set at $100 \%$. Angular selectivity curves have been produced using these parameters for the three different thicknesses used in this study $(50 \mu \mathrm{m}, 75 \mu \mathrm{m}, 100 \mu \mathrm{m})$ and are presented in Figs. (4a-4c). The modelled curves follow the expected trends with a narrowing angular selectivity as thickness is increased, from a FWHM of $\approx 24^{\circ}$ for the $50 \mu \mathrm{m}$ case shrinking to $\approx 12^{\circ}$ for the $100 \mu \mathrm{m}$ case. All curves have an obvious peak, centred close to $0^{\circ}$ due to the low spatial frequency.

These angular selectivity curves can be compared to the closest corresponding data for the diffuser samples (diffuser efficiency graphs over a range of input angles for the strongest diffusers at the matching thicknesses). The data has been obtained using the setup in Fig. 2a over a $50^{\circ}$ span with a $0.25^{\circ}$ step-size and processed with 
a moving average filter over 7 points. Examples of the corresponding experimental data for $0.5 \mathrm{~mL}, 0.75 \mathrm{~mL}$ and $1 \mathrm{~mL}$ layers are shown in Figs. (4d-4f). The experimental curves display a different set of characteristics to the modelled data, for example, within the $50^{\circ}$ span there is no obvious FWHM due to the large width of the curves in all cases. In addition there is no obvious single peak, with a more plateau shaped maximum evident instead. Finally in the cases of the $0.75 \mathrm{~mL}$ and $1 \mathrm{~mL}$ layers, where the diffuser efficiency approaches $100 \%$ at its peak, it remains $>70 \%$ even at the extremes of the incident angles indicating a different curve shape than is predicted by KCWT.

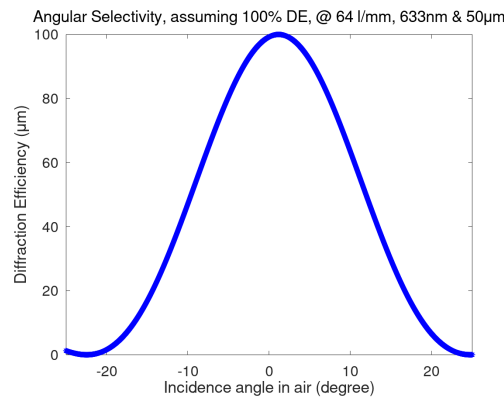

(a)

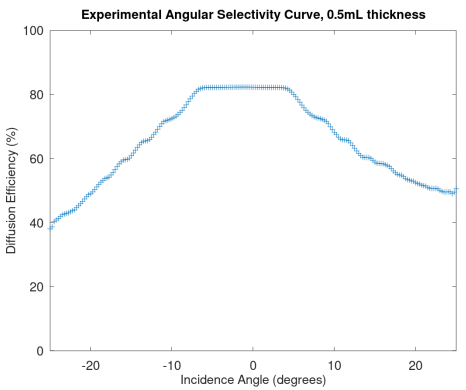

(d)

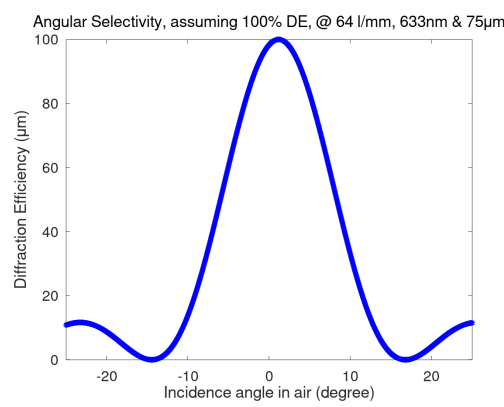

(b)

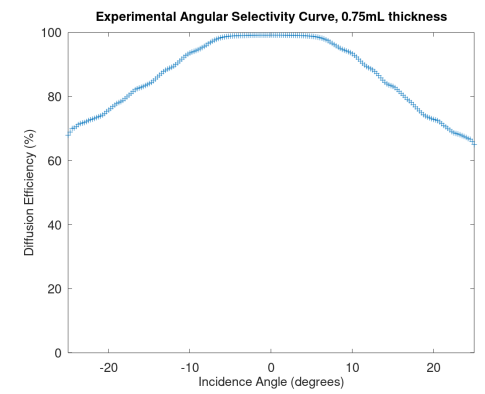

(e)

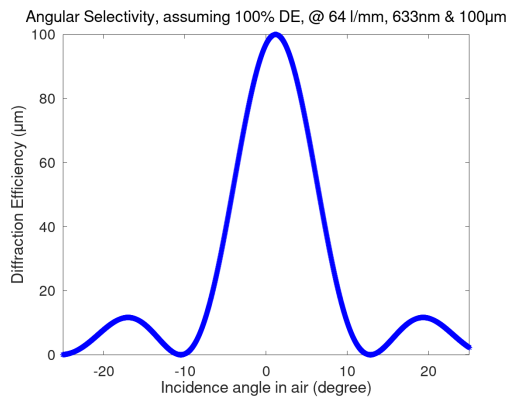

(c)

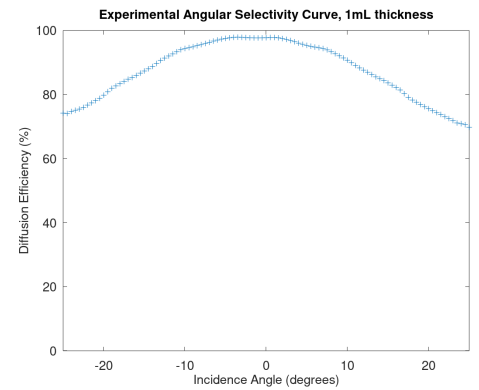

(f)

Figure 4: Angular selectivity curves modelled using KCWT for $50 \mu \mathrm{m}, 75 \mu \mathrm{m}$ and $100 \mu \mathrm{m}$ thick layers (a-c) and experimental diffuser efficiency curves for $0.5 \mathrm{~mL}, 0.75 \mathrm{~mL}$ and $1 \mathrm{~mL}$ layers $(\mathrm{d}-\mathrm{f})$

\section{RESULTS}

\subsection{Recording Conditions Optimisation}

Holographic recording conditions optimisation was carried through the variation in exposure intensity and exposure time delivered to the photosensitive sample. Combined these factors vary the exposure energy delivered to each sample. In addition three different thickness of photopolymer layer were tested with a range of exposure energies.

Initially a study of the variation in recording power was carried out for a single layer thickness (corresponding to a $0.75 \mathrm{~mL}$ volume of photopolymer solution deposited, onto a glass slide). The exposure intensity was varied from $0.75 \mathrm{~mW} / \mathrm{cm}^{2}$ to $1 \mathrm{~mW} / \mathrm{cm}^{2}$ to $1.25 \mathrm{~mW} / \mathrm{cm}^{2}$. For each recording intensity diffusers with a range of exposure times were recorded, all within exposure energy bounds of $30-120 \mathrm{~mJ} / \mathrm{cm}^{2}$. The peak diffuser efficiency for each recording condition was plotted against the exposure energy and the results are presented in Fig. 5. For each point three diffusers samples were recorded and the mean and standard deviation are shown. Fig. 5a is for a recording intensity of $0.75 \mathrm{~mW} / \mathrm{cm}^{2}$, Fig. $5 \mathrm{~b}$ is for a recording intensity of $1 \mathrm{~mW} / \mathrm{cm}^{2}$ and Fig. $5 \mathrm{c}$ is for a recording intensity of $1.25 \mathrm{~mW} / \mathrm{cm}^{2}$. All of the results are plotted together in Fig. $5 \mathrm{~d}$.

From Fig. 5 a number of trends can be observed. As expected, from previously published work, ${ }^{13}$ delivering the same amount of exposure energy using different recording intensities results in different diffuser efficiency values. Essentially the higher the recording intensity the quicker diffuser efficiency jumps to a high level (> $85 \%$ ). However the maximum diffuser efficiency value reached does not depend linearly on recording intensity. 
Both of these effects are due to the manner in which the photopolymer records diffractive features within its volume. Through both polymerisation of monomers in the bright regions and diffusion of both monomers and short polymer chains from the dark regions into the bright regions a refractive index modulation occurs. The optimal balance of recording conditions must be struck to allow these two effects (particularly the diffusion of monomers/short polymers) contribute to the desired refractive index change in the layer.

In Fig. 5 it can be seen that all three recording intensities $\left(0.75 \mathrm{~mW} / \mathrm{cm}^{2}, 1 \mathrm{~mW} / \mathrm{cm}^{2}\right.$ and $\left.1.25 \mathrm{~mW} / \mathrm{cm}^{2}\right)$ reach very high levels of diffuser efficiency, $>97 \%$ (Fig. $5 \mathrm{~d}$ ). This occurs in the $90-110 \mathrm{~mJ} / \mathrm{cm}^{2}$ exposure energy range. However the $1 \mathrm{~mW} / \mathrm{cm}^{2}$ (Fig. 5b) reaches a slightly higher final value than the other two and does so with less overall variability. For this reason the $1 \mathrm{~mW} / \mathrm{cm}^{2}$ recording intensity is chosen as the best power conditions to try and optimise the thickness for.

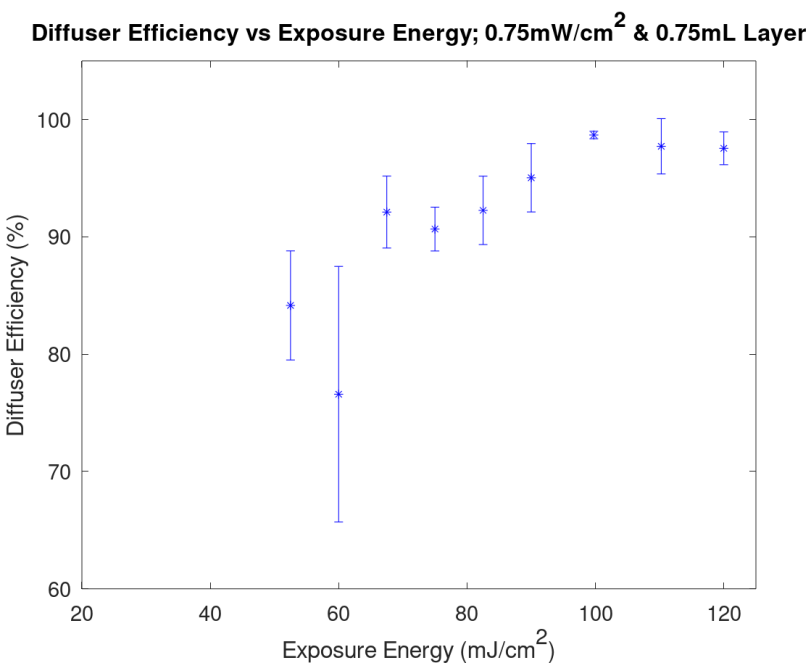

(a)

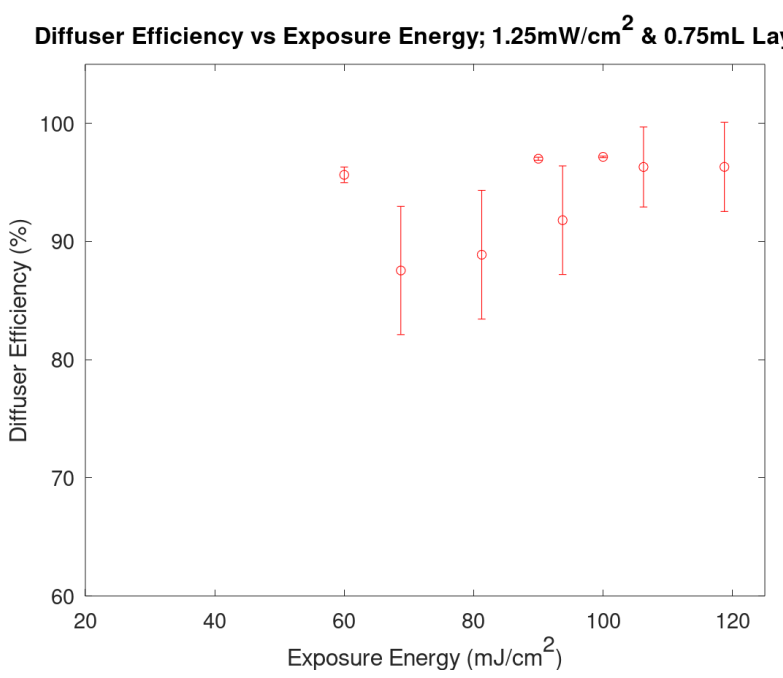

(c)

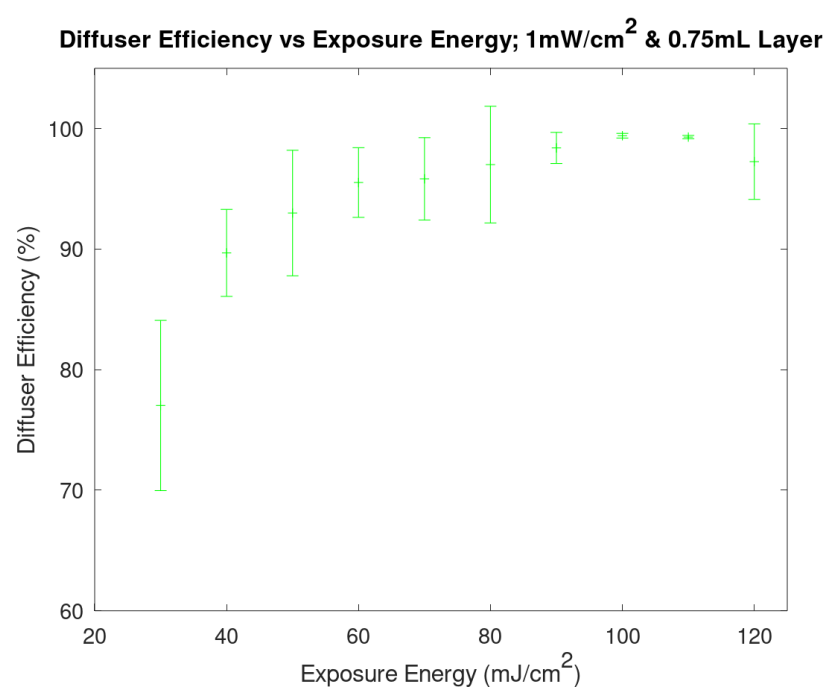

(b)

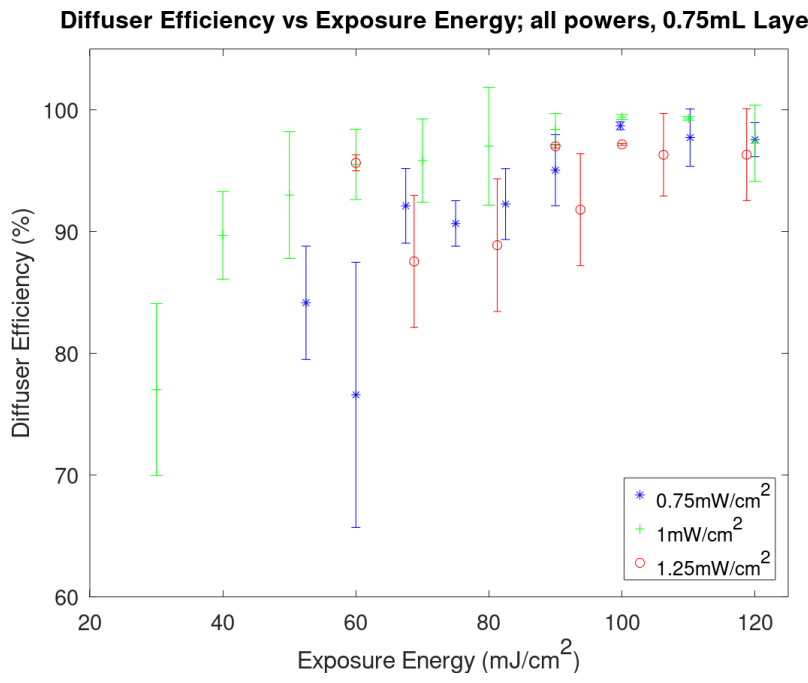

(d)

Figure 5: Diffuser efficiency results for varying recording intensity and exposure energy for a $0.75 \mathrm{~mL}$ layer; recording intensity $=0.75 \mathrm{~mW} / \mathrm{cm}^{2}(\mathrm{a})$, recording intensity $=1 \mathrm{~mW} / \mathrm{cm}^{2}(\mathrm{~b})$, recording intensity $=1.25 \mathrm{~mW} / \mathrm{cm}^{2}$ (c) and all recording intensities (d).

A study of the variation of layer thickness for a single recording intensity $\left(1 \mathrm{~mW} / \mathrm{cm}^{2}\right)$ was then carried out. Three layer thickness were used corresponding to three different volumes of photopolymer solution deposited, 
$0.5 \mathrm{~mL}, 0.75 \mathrm{~mL}$ and $1 \mathrm{~mL}$ ). For each thickness diffusers with a range of exposure times were recorded to vary exposure energy within the bounds of $30-120 \mathrm{~mJ} / \mathrm{cm}^{2}$. The peak diffuser efficiency for each recording condition was plotted against the exposure energy and the results are presented in Fig. 6. For each point three diffusers samples were recorded and the mean and standard deviation are shown. Fig. 6a is for a layer thickness corresponding to $0.5 \mathrm{~mL}$, Fig. $6 \mathrm{~b}$ is for a layer thickness corresponding to $0.75 \mathrm{~mL}$ and Fig. $6 \mathrm{c}$ is for a layer thickness corresponding to $1 \mathrm{~mL}$. All of the results are plotted together in Fig. 6d.

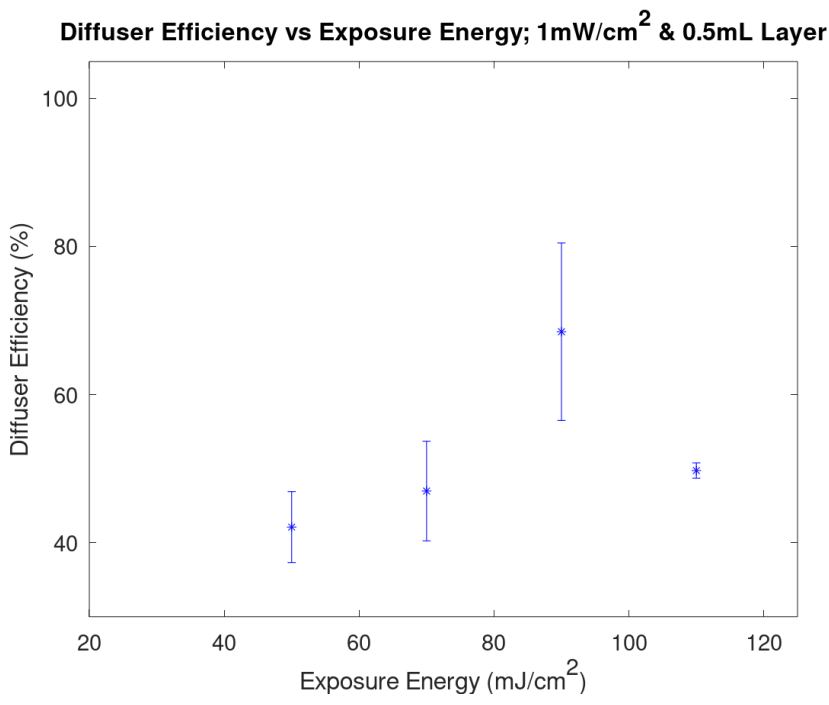

(a)

Diffuser Efficiency vs Exposure Energy; $1 \mathrm{~mW} / \mathrm{cm}^{2} \& 1 \mathrm{~mL}$ Layer

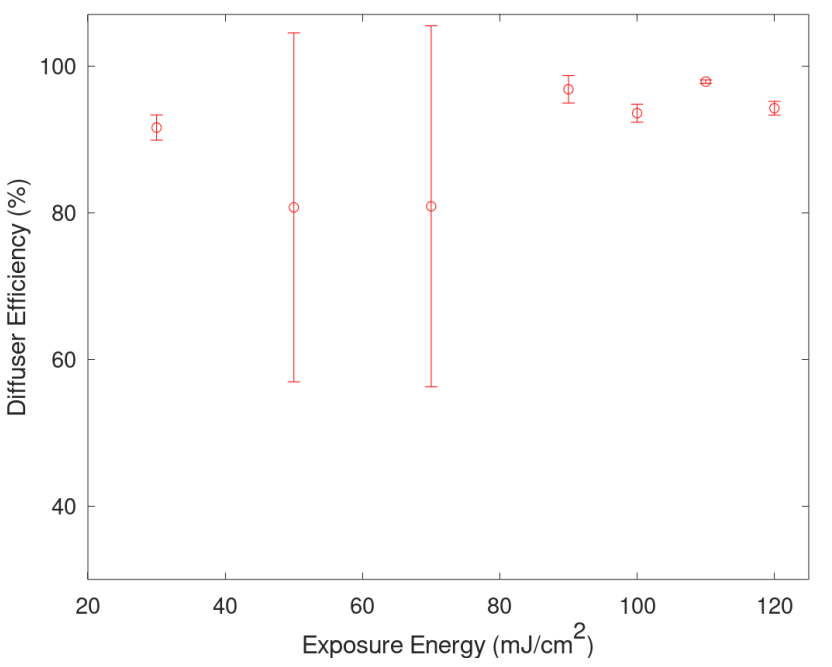

(c)

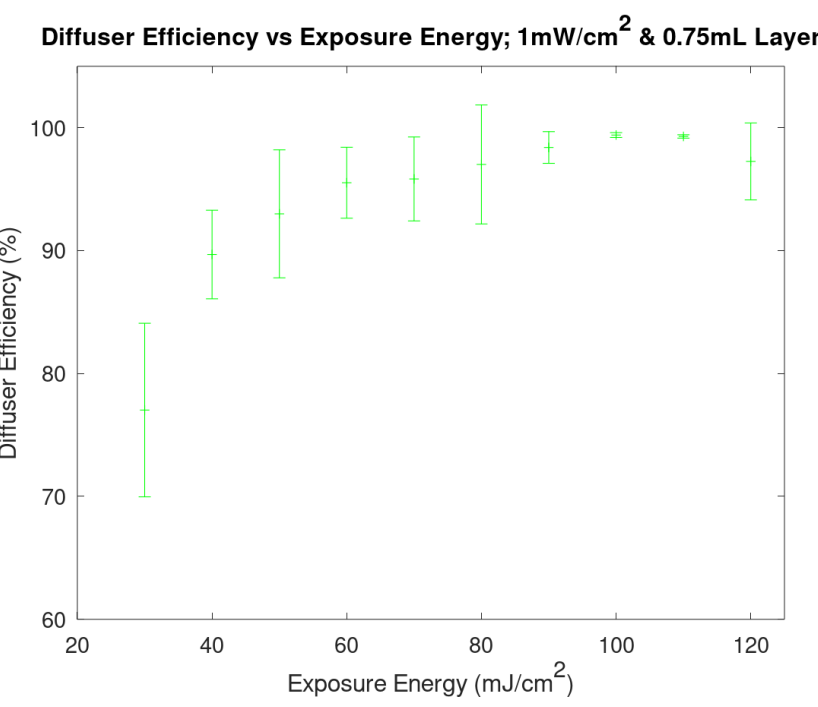

(b)

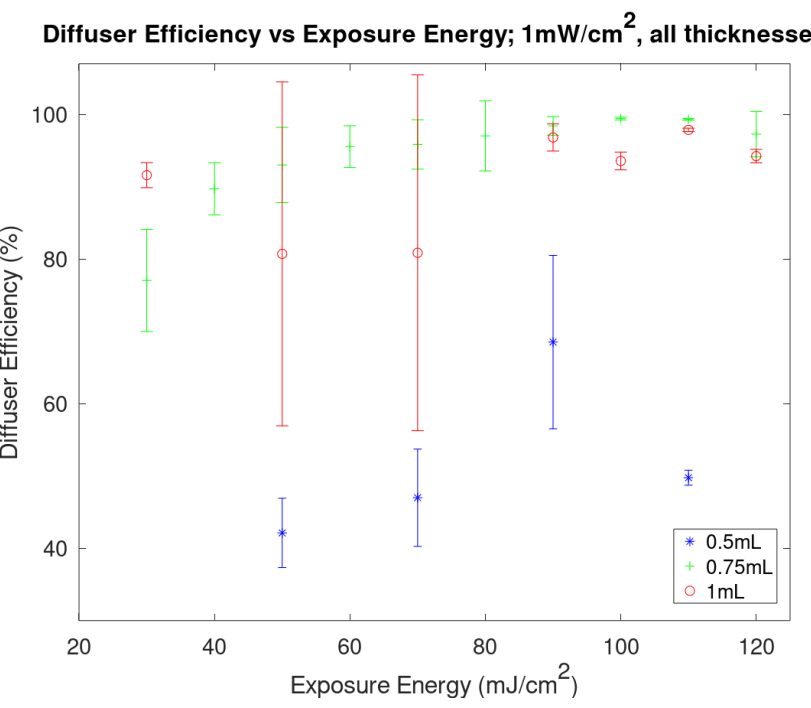

(d)

Figure 6: Diffuser efficiency results for varying layer thickness and exposure energy for a $1 \mathrm{~mW} / \mathrm{cm}^{2}$ recording intensity; layer thickness $=0.5 \mathrm{~mL}(\mathrm{a})$, layer thickness $=0.75 \mathrm{~mL}(\mathrm{~b})$, layer thickness $=1 \mathrm{~mL}(\mathrm{c})$ and all layer thicknesses (d).

From Fig. 6 some results are immediately obvious, for example thin layers (Fig. 6a) struggle to perform well in terms of diffuser efficiency. No exposure energy gives an average $>70 \%$ and there is no increase in diffuser efficiency with increasing exposure energy. This may be due the shorter path length for any phase change to occur or to the smaller amount of photosensitive dye available to induce the photochemical processes, or both. Another aspect of note is the seemingly large variation in some exposure energy values for the $1 \mathrm{~mL}$ case (Fig. $6 \mathrm{c}$ ). 
The two large errorbars are due to outlier data, with low diffuser efficiency, that pull the average considerably lower; without these outliers the points correspond to the rest of the trend evident. It is possible there was an outside effect preventing a good recording of the diffusers for these samples (e.g., air bubbles during lamination or excessive movement during recording). However as no inherently erroneous aspect was noted during the experiments the results from these samples are shown here for completeness.

Fig. 6d highlights how layer thickness affects the diffuser efficiency, with a thicker layer $(1 \mathrm{~mL})$ giving consistently strong results from low exposure energies $\left(30 \mathrm{~mJ} / \mathrm{cm}^{2}\right)$ through to high exposure energies $\left(120 \mathrm{~mJ} / \mathrm{cm}^{2}\right)$. Thin layers $(0.5 \mathrm{~mL})$ are not suitable for fabricating strong diffusers. In this study the highest value diffuser efficiency was found for the condition of $0.75 \mathrm{~mL}$ layers, with a recording intensity of $1 \mathrm{~mW} / \mathrm{cm}^{2}$ and an exposure energy of $100 \mathrm{~mJ} / \mathrm{cm}^{2}$. However as a thicker layer of $1 \mathrm{~mL}$ gives similar results (with often overlapping error bars) it may be more suitable in mass manufacture to achieve consistently good results, with lower exposure energies/times.

\subsection{Diffuser Properties}

A comparison of the beam-shape outputted from the strongest diffusers produced in this study to other relevant diffusers is mapped in Fig. 7. This was performed using the setup in Fig. 2b over a range of $30^{\circ}$, with an angular step size of $0.25^{\circ}$. In Fig. 7 the intensity of the detected light is plotted on a log scale. The beam profile output of the probe laser diode (without passing through any samples) is shown in green as a control. The black and yellow lines represent ground glass diffusers of 120 grade grit polish (considered a coarse diffuser) and 660 grade grit polish (considered a fine diffuser) respectively. The performance of the strongest diffuser previously recorded and published using this method ${ }^{8}$ is illustrated in red. The blue line represents the averages of the three samples of strongest diffuser produced in this study $\left(0.75 \mathrm{~mL}\right.$ thick layer, recording intensity $=1 \mathrm{~mW} / \mathrm{cm}^{2}$, exposure energy $\left.=100 \mathrm{~mJ} / \mathrm{cm}^{2}\right)$. All data has been processed using a moving average filter over 5 points.

The ground glass diffusers show a beam-shape with a very strong decrease in the zero order and a spreading of the transmitted light over a wide angle, with an irregular pattern. In comparison the previous strongest diffuser (in red) shows a pronounced zero order overlaying the beam shape of its diffused output. The optimised HOE diffuser produced here shows a much reduced zero order (by a factor of five), compared with previously. It also maintains the desired beam shape, with the zero order merging into a smooth cone beam (unlike the wide and irregular spread of the ground glass diffusers or the spiky zero order seen in the previously published holographic diffusers).

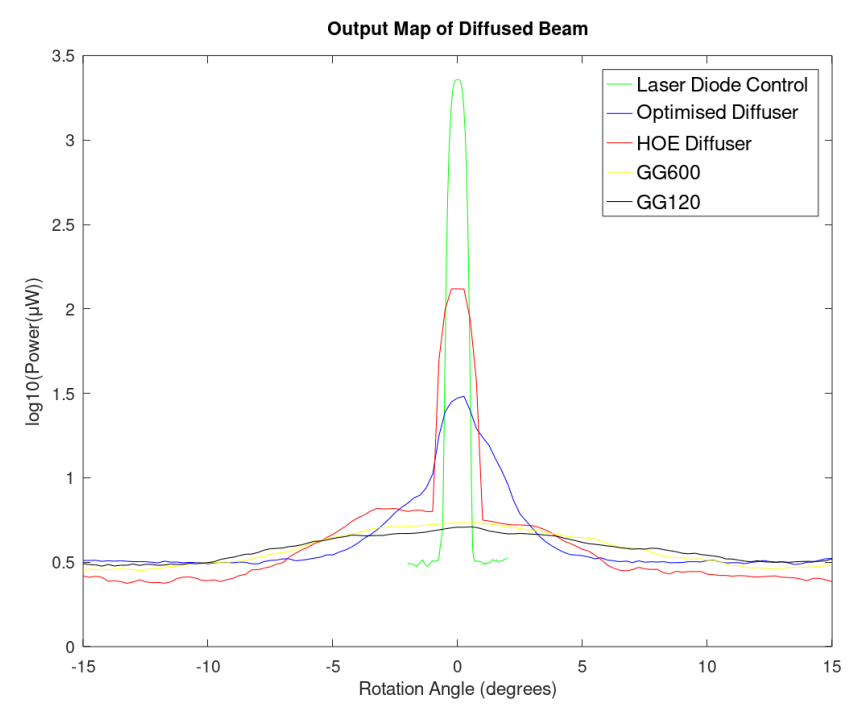

Figure 7: Beam shape output map of various diffusers (log-scale intensity) showing; Laser diode control (green), ground glass diffusers (black and yellow), previous strongest diffuser (red) and optimised diffusers (blue). 


\section{CONCLUSIONS}

In this work it has been shown that the zero order present in volume holographic diffusers, fabricated using a direct recording of a controlled speckle pattern, can be significantly reduced by a factor of five. This brings the remaining zero order down to $\approx$ level found in ground glass diffusers. This is done while retaining the desired diffused beam output characteristics. The zero order is suppressed through varying the recording conditions (recording intensity $\left[0.75-1.25 \mathrm{~mW} / \mathrm{cm}^{2}\right]$, layer thickness $[\approx 50-100 \mu \mathrm{m}]$, exposure time $[30-180 \mathrm{~s}]$ and exposure energy $\left[30-120 \mathrm{~mJ} / \mathrm{cm}^{2}\right]$ ) delivered to the sample to successfully optimise the strength of the diffusers. This optimisation should be done for each speckle size and each recording material in order to produce the best results.

Modelling of the diffraction through the diffusers was also attempted using Kolegnik Coupled Wave Theory, which proved to be an inappropriate approach to model diffusers with such characteristics. KCWT predicted a much narrower FWHM than were observed experimentally (less than half), which could be due to the wide range of spatial frequencies present in the diffuser structure or as a result of being at the edge of the "thick" regime as indicated by the Q parameter. Future work will look at exploring other modelling diffraction grating theories and methods.

\section{ACKNOWLEDGMENTS}

CJ carried out the experimental work presented; KM co-designed the experimental work, performed data analysis and drafted the manuscript; SK carried out the modelling work; DC co-designed the experimental work, interpreted data and edited the manuscript; SM contributed to the modelling work, interpreted data and edited the manuscript. The authors wish to acknowledge the FOCAS Research Institute for use of facilities and equipment.

Funding sources (KM): Enterprise Ireland (IP20190855), co-funded under the EU European Regional Development Fund (ERDF) 2014-2020 Programme.

\section{REFERENCES}

[1] V. Toal, [Introduction to Holography], CRC Press (2011).

$[2]$ E. N. Leith and J. Upatnieks, "Reconstructed Wavefronts and Communication Theory," JOSA A 52(10), 1123-1130 (1962).

[3] S. I. Kim, Y. S. Choi, Y. N. Ham, C. Y. Park, and J. M. Kim, "Holographic diffuser by use of a silver halide sensitized gelatin process," Appl. Opt. 42(14), 2482-2491 (2003).

[4] R. Ahmed, A. K. Yetisen, A. El Khourya, and H. Butt, "Printable ink lenses, diffusers, and 2D gratings," Nanoscale 9, 266-276 (2017).

[5] M. L. Piao, K. C. Kwon, H. J. Kang, K. Y. Lee, and N. Kim, "Full-color holographic diffuser using timescheduled iterative exposure," Appl. Opt. 54(16), 5252-5259 (2015).

[6] S. Wadle, and R. Lakes, "Holographic Diffusers: Diffusers with low backscatter," J. Mod. Opt. 42, 1387-1396 (1995).

[7] J. Park, J. Y. Cho, C. Park, K. Lee, H. Lee, Y. H. Cho, and Y. Park, "Scattering Optical Elements: Stand-alone optical elements exploiting multiple light scattering," ACS Nano 10, 6871-6876 (2016).

[8] K. Murphy, V. Toal, I. Naydenova, and S. Martin, "Holographic beam-shaping diffractive diffusers fabricated by using controlled laser speckle," Opt. Express 26, 8916-8922 (2018).

[9] S. Martin, P. Leclere, Y. Renotte, V. Toal, and Y. Lion, "Characterization of an acrylamide-based dry photopolymer holographic recording material," Opt. Eng. 33, 3942-3946 (1994).

[10] D. Vather, et al, "Serialized holography for brand protection and authentication," Appl. Opt. 57, E131-E137 (2018)

[11] H. Kogelnik, "Coupled wave theory for thick hologram gratings", The Bell System Technical Journal, 48, 2909-2947 (1969).

[12] S. K. Keshri, K. Murphy, I. Naydenova and S. Martin, "Theoretical Analysis of a Volume Holographic Lens Using Matlab," in [2019 PhotonIcs 83 Electromagnetics Research Symposium - Spring (PIERS-Spring)], 2814-2818 (2019).

[13] S. Keshri, K. Murphy, V. Toal, I. Naydenova, and S. Martin, "Development of a photopolymer holographic lens for collimation of light from a green light-emitting diode," Appl. Opt. 57, E163-E172 (2018) 